\title{
DYNAMIC MONITORING METHOD OF ILLEGAL BUILDINGS USING SPATIOTEMPORAL BIG DATA BASED ON URBAN HIGH LYING ZONES
}

\author{
Baishou $\mathrm{Li}^{1,2}$, Zhaocheng Tan ${ }^{1}$ \\ ${ }^{1}$ College of Geomatics and Geoinformation, Guilin University of Technology, Guilin, Guangxi, China, \\ (lbszhb,tanzhaocheng1998@163.com) \\ ${ }^{2}$ Guangxi Key Laboratory of Spatial Information and Geomatics, Guilin, Guangxi, China, \\ (lbszhb,tanzhaocheng1998@163.com) \\ Commission VI, WG VI/4
}

KEY WORDS: Spatiotemporal Big Data, Sensor Network, Hardware and Software, Illegal Buildings, Dynamic Monitoring

\begin{abstract}
:
China's urban illegal buildings are emerging in an endless stream with a large number. There is a wide demand for urban illegal buildings monitoring in urban management departments, including Beijing, Shanghai, Guangzhou and other regions where urban management is facing increasingly serious problems of illegal buildings. It is urgent to solve the common problem of "urban disease" caused by urban illegal buildings, and a new automatic monitoring method that can reduce the cost of urban management is urgently needed. This kind of automated monitoring method of illegal buildings has a wide market demand in Urban Management and Law Enforcement and the Ministry of Land and Resources. Existing technologies cannot realize long-term, autonomous, rapid and intelligent dynamic real-time monitoring of urban illegal buildings, which leads to the problem that illegal buildings' behaviors cannot be stopped in time. There is a possibility to solve these problems using the ubiquitous network of base stations in cities to monitor illegal buildings.
\end{abstract}

This paper proposes a dynamic monitoring method of illegal buildings using spatiotemporal big data based on urban high lying zones. Through the Spatiotemporal sensor network technology, the tilt-type stereo camera is set up at the high lying zones around the survey area. According to the real-time ambient temperature and humidity numerical data fed back by the temperature and humidity sensor, the tilt-type stereo camera uses intelligent time-lapse photogrammetry technology to obtain multiple stereo pairs. The tilted remote sensor transmits the multiple stereo pairs to the urban dynamic analysis service network using wireless transmission. The urban dynamic analysis service network will complete a series of analysis and processing operations without any human intervention, and then transmit the results of the analysis to the early-warning terminal successively through the base station, WIFI and other wireless transmission methods. Finally, the illegal building data is uploaded to the terminal. That is to say, the urban dynamic analysis service network can realize the intelligent, automatic analysis and processing of spatial analysis server and the operation of calling all database data and storing data.

The method proposed in this paper uses the high lying zones around the survey area to expand the monitoring range, improve the accuracy of monitoring data, realize long-term real-time monitoring, and fully utilize the characteristics of Spatiotemporal sensing network technology intelligent, autonomous, wireless transmission, etc., significantly reducing labor. It greatly shortens the process from the emergence to the discovery of illegal buildings in cities. The workload of monitoring has improved the efficiency of dynamic monitoring and warehousing of illegal buildings data in cities.

\section{INTRODUCTION}

With the rapid development of China's economy, the city's appearance is changing with each passing day. At the same time, the problem of illegal construction in certain areas of the city has become increasingly prominent. The problems of private construction, construction, expansion , and expansion are common and repeated. In order to grasp the changes of the city and achieve effective management, the planning department urgently needs to grasp the real-time information of the illegal geographical coordinates of the city and the illegal land area ${ }^{[1]}$. The traditional monitoring and management of illegal buildings are mainly based on the reports and confirmations of the masses. The illegal buildings are repeatedly prohibited and cannot be discovered in time. In the demolition work, it often reveals that there is insufficient communication between the urban construction law enforcement department and the planning department. The planning department failed to timely report the permission information of the building to the urban construction law enforcement department. In addition, the urban construction law enforcement department cannot determine and timely grasp the situation of illegal building construction ${ }^{[2]}$. The emergence of the UAV remote sensing platform has provided a new technical approach for the national geographic condition monitoring, especially for illegal building monitoring. UAV remote sensing has the characteristics of mobility, flexibility, and security, and can obtain high-resolution, multi-view remote sensing images, but it also has the disadvantages of high cost and long data update period ${ }^{[3]}$. However, there are ubiquitous base stations in cities such as Beijing, Shanghai, and Guangzhou. We can make it possible to monitor illegal buildings by setting up multiple sensors at high grounds in the city ${ }^{[4]}$. 


\section{METHOD FOR MONITORING ILLEGAL BUILDINGS IN TRADITIONAL CITIES}

Data acquisition through traditional aerial photography (satellite remote sensing, aerial remote sensing) is not only costly, has a long cycle, and is not suitable for short-term high-frequency urban monitoring ${ }^{[1]}$.

At present, the data sources of urban illegal building monitoring methods are mainly: UAVs and satellite remote sensing images. However, the method of illegal building monitoring based on these two data sources has certain limitations. For example, the methods of illegal building monitoring based on drones mainly include: (1) The flight of the drone is subject to air traffic control and needs to be reported to the relevant department in advance, resulting in a longer cycle of acquiring image data and the height and range of flight are also subject to strict restrictions [1];(2) drone flight requires the use of professional flying hand control, resulting in high labor costs ${ }^{[3]}$; (3) Due to the limited endurance of the drone, multiple flights are required to complete a wide range of image data collection ${ }^{[3]}$; (4) Most of the image data collected by the UAV is stored in the memory card, so that the analyst needs to import the data collected by the UAV into the computer for processing and analysis. The degree of automation is low, and it will extend the cycle of discovering illegal buildings ${ }^{[5]}$.

Secondly, the methods of illegal building monitoring based on satellite remote sensing images mainly include: (1) The long-term update period of satellite remote sensing images is mainly due to the fact that the long revisit period of satellites is not conducive to the timely updating of illegal construction data ${ }^{[6]}$. (2) The collected image data of the survey area has different levels of cloud coverage, which may result in the quality of the collected image data cannot be guaranteed ${ }^{[7]}$. (3) The price of satellite remote sensing image is very expensive, which is not conducive to the high frequency of the survey area ${ }^{[8]}$. (4)The spatial resolution of satellite image data does not meet the monitoring requirements for illegal buildings, which means that the subtle changes in illegal buildings are not obvious on satellite remote sensing images ${ }^{[9]}$.

In addition, at present, most illegal building monitoring methods based on UAVs and satellite remote sensing images have a low degree of automation, and artificially identify the massive image data acquired by them to determine illegal buildings and these methods cannot provide early warning of the discovery and progress of illegal buildings ${ }^{[10]}$. Based on the research and analysis of the defects of most illegal building monitoring methods on the market, we propose a dynamic monitoring method for urban illegal buildings using high ground points. The method is characterized by low cost, high efficiency, high frequency and dynamic monitoring of illegal buildings in the city.

In view of the deficiencies of the prior art, the method proposes a dynamic monitoring method of illegal buildings using spatiotemporal big data based on urban high lying zones, it making full use of the urban terrain high point, and improving the monitoring range of the illegal building dynamic monitoring method. It meets the requirements for building stocks to be updated on weekdays or days. The power supply equipment meets the power supply requirements in areas where it is difficult to erect electric lines such as mountains and solves the key problems of small-scale and high-cost monitoring of illegal buildings in traditional cities.it realizes the dynamic monitoring of illegal buildings in cities. At the same time, it meets the real-time requirements of urban illegal building monitoring "once the illegal building appears, it will be immediately discovered and stopped".

\section{THE TECHNICAL PRINCIPLE OF THIS MONITORING METHOD}

The method adopts a Spatiotemporal sensing network technology, and the tilt-type stereo camera is set up at the high lying zones around the survey area. According to the real-time ambient temperature and humidity numerical data fed back by the temperature and humidity sensor, the tilt-type stereo camera uses intelligent time-lapse photogrammetry technology to obtain multiple stereo pairs. The tilted remote sensor transmits the multiple stereo pairs to the urban dynamic analysis service network using wireless transmission. It generates the measurement area DSM based on the stereo image pair ${ }^{[11]}$, and detects the changed feature based on the three-dimensional incremental analysis ${ }^{[12]}$, adopting multi-scale Segmentation algorithm, accurate DEM model and DSM model extract and identify buildings ${ }^{[10]}$, use Arcgis to statistically analyze the polygons of the monthly change area of the building, and judge the type of change based on DEM model and DSM model, refer to the building of the Bureau of Housing and Construction Approving the map database to obtain attribute information, using ERDAS to quantitatively extract illegal building height and area and depositing in the illegal building database of the construction bureau. In addition, this method can realize real-time warning of illegal buildings, that is, illegal building data is sent to the handheld terminal through warning, and provides real-time illegal building information for law enforcement agencies ${ }^{[13]}$. It should be noted that the building data obtained after the analysis by the tilted camera is transmitted to the spatial analysis server through wireless transmission such as WIFI, and then compared with the planning approval building data in the planning approval database server. Illegal building data avoids the complicated process of manually importing computer analysis, greatly improves the efficiency of information transmission, and further guarantees the function of this method for real-time monitoring of illegal buildings.

\section{TECHNICAL SPECIFIC PROCESS OF THIS MONITORING METHOD}

(1)Selection of ground platform remote sensor: According to the spectral, radiation measurement and geometric characteristics, the performance of the remote sensor is fully considered, and the stereo mapping camera is selected. Based on the strict requirements of the stereo image pair to generate the DSM and 3D incremental analysis in the geometric accuracy of the imaging quality, the RCD30, Midas, SWDC- 
5, and the tilting camera AP2600, which meet the geometric accuracy requirements of the imaging quality, are preliminarily selected. By comparison, the selection scheme selects the AP2600, which is a compact design of the twocamera integrated compact design with high positioning accuracy (image resolution up to $2 \mathrm{~cm}$ ), high photography efficiency $(46 \%$ higher than the traditional five-camera efficiency) and low cost. The ultimate choice of remote sensors.

(2) Visual domain analysis to develop a system erection plan: use the Arcgis "terrain to TIN" geoprocessing tool to establish a triangulated irregular network (TIN). According to the accurate digital topographic map and its DEM in the mountainous area, the Arcgis view analysis, simulation observation angle and resolution are used to determine the coverage orientation and field angle range of the mountain, and the remote sensing device erection position is selected. The visual area of the monitoring area is obtained by using a visible field, a Thiessen Polygon, and the like.

In order to more objectively understand the visual field status of the site monitoring area and provide a basis for the site optimization criteria, the method uses the monitoring area monitoring rate indicator to measure: Monitoring area monitoring rate is the ratio of the visible field in the monitored area of all affected monitoring points to the area of the area.

(3) Construction and erection of urban illegal building dynamic monitoring system:

Ground platform remote sensor erection: After the temperature and humidity sensor is matched with the photographic camera, the platform is erected according to the optimal monitoring point analyzed in step (2), and the camera is used to fix the bracket in a specific orientation and height according to the measurement needs.

Construction of power supply equipment: Based on the current industrialization of solar photovoltaic power generation technology, this method intends to use solar photovoltaic power generation technology to supply power to the remote sensors. It will solve the problem that the conventional power line power supply line is difficult to set up, the cost is high, and the power supply of each remote sensor is strong and easy to generate even pieces of electricity. Compared with conventional power line power supply, solar photovoltaic power generation equipment can operate normally under normal temperature illumination. And it has no special requirements for the flatness of the site. It is suitable for the high point of the mountainous area. It has the advantage of being able to store energy and provide continuous and independent power supply for each remote sensor.

The temperature and humidity sensor is installed at the monitoring point to detect and acquire the temperature and humidity data of the measuring point environment, and the temperature and humidity data is transmitted to the urban dynamic monitoring service network by the communication base station by wireless communication, and the urban dynamic monitoring service network controls the data collection under the optimal temperature and humidity conditions of the photographic camera through the communication base station according to the real-time temperature and humidity data of the survey area.

(4) Remote sensor parameter setting: This method considers that urban buildings cannot be changed greatly in one day due to subjective and objective reasons, and one week ( 7 days) is selected as the observation period. Measurement time period: This method takes into account the influence of light, temperature ,and humidity on the measurement, and the influence degree of each factor is different at different time periods of the day. Avoiding no radiation sources at night, sensor noise caused by an excessively strong source of 12-14 points can cause local over-bright noise in the image. Therefore, according to the stability analysis of the radiance value of the radiation statistical change detection area, the time (16-18 points) of the location of the hill is selected as the main detection period.

Adjusting the attitude of the remote sensor before measurement: The tilting camera AP2600 is integrated by two rotating cameras. Since the orientation elements in the camera installation and debugging phase will produce some residual phase difference, which leads to large optical distortion, it is necessary to Strictly calibrated before aerial photography. Through the calibration to obtain the camera's orientation elements and various distortion parameters, using the Space Resection model to check the camera, to calculate the camera's internal and external orientation elements and distortion parameters (camera focal length, principal point coordinates, radial distortion, Eccentric distortion).

(5) Using the temperature and humidity sensor to detect whether the external environment meets the measurement conditions: the change of the external temperature has a great influence on the imaging quality of the remote sensor. The ideal working temperature of the remote sensor is $-20 \sim 40$ degrees Celsius. The change in temperature causes the refractive index of the optical element and the refractive index of the air, the thickness of the optical element and the radius of curvature, and the air gap between the optical elements to change, thereby changing the optical path of the beam transmitted through the optical system, and thus the focal length of the system changes. The change of the focal length of the optical system causes the ideal imaging surface to deviate from the photosensitive surface of the receiver, causing the system to defocus, causing the image point to form a speckle on the image receiving surface, which directly leads to a decrease in image quality. In an environment where the relative humidity reaches $60 \%$ or more, the surface of the remote sensor lens is prone to produce undetectable mildew affecting the image quality. Therefore, in order to ensure that the remote sensor works in an environment of -20 40 degrees Celsius and less than $60 \%$ relative humidity, it is necessary to match the temperature and humidity sensor to the remote sensor and specify a temperature of $-20 \sim 40$ degrees Celsius and relative humidity of less than $60 \%$. Under normal working conditions, the temperature and humidity sensor detects the measurement point environment in real-time. When the outside temperature is too high and the internal temperature of the remote sensor rises to a dangerous value, the temperature and humidity sensor issues a warning and stops the photogrammetry of the remote sensor.

(6) Oblique photogrammetry to obtain stereo pairs: Referring to Figure 2, a remote sensor mounted at a high ground level simultaneously captures urban building images from two different angle sub-cameras. The AP2600's built-in multicameras unified control is $46 \%$ more efficient than the traditional five-camera. The two-camera remote sensor captures $60 \%$ to $53 \%$ of the photos with overlapping degrees 
and then generates a stereo image of the monitoring area with a parallax greater than 6 degrees by internal processing. (7) Automatic sharpness processing such as stereo pair enhancement: In the process of acquisition, transmission , and encoding, the image will inevitably be interfered by noise, and the existence of noise will cause difficulties in image observation, processing , and interpretation.

We use spatial domain linear transform enhancement processing, image filtering can minimize or even completely filter out the noise in the image, to improve image quality, improve the understanding of the image and facilitate the subsequent higher-level processing and application purposes. (8) Generating the DEM and DSM based on the stereo image pair: This method uses the SuperMap iDesktop8C to set the high-precision raster dataset produced by the map range, resolution, and camera height to quickly extract the oblique photography model and the bottom surface of the building vector to generate the DEM, DSM. The specific steps are as follows: generate a tilt photography profile, create a new plane scene, add the generated configuration file to the scene, generate a DSM in 3D analysis, set the resolution and camera height parameters and then use the DEM function in spatial analysis to build the DEM.

(9) Establish a building inventory database: See Figure 3. Use the spatial database engine (ArcSDE) and the database management system (SQL Server) to build a building inventory database. In order to facilitate spatial analysis, the database must have the ability to store building geographic coordinate class spatial data and building change types, construction time class attribute data. The spatial data structure is divided into a vector data structure and a raster data structure, and the attribute table stores attribute data, and the spatial data and the attribute data are mutually determined by a unique code.

(10) Surface change detection based on three-dimensional incremental analysis: The three-dimensional geographic information is converted from the DXF format by vectorization, vector data stitching, spatial correction to SHP file and then ArcMap statistics is used to calculate the area of the building. Referring to Figure 4, the spatial superposition analysis of the two-stage DSM model is carried out by ArcScene, and ArcScene's ArcToolBox superposition analysis function is used to analyze the dynamic changes of all features in the horizontal and vertical directions from different regional functions and spatial locations.

(11) Extraction of changing buildings: Extracting changing building information from the DSM. Due to the diversity of the shape of the building and the complexity of the terrain, the extraction of the building is very difficult. It is very difficult to extract buildings from high-resolution images only, so this method proposes to extract non-ground points using accurate DEM models and DSM models:

$$
S=D S M-D E M
$$

Where $\mathrm{S}$ is a non-ground point. Two types of points are separated by means of DSM: 1.ground point and noise point, 2.consisting of buildings, vegetation, and shadows to form non-ground points. Considering the complexity between vegetation and buildings, we use the multi-scale method to divide it twice, and each polygon represents a kind of ground object, and then it is secondarily classified by spectral characteristics and texture information.

Since the texture characteristics are spatial variations and repetitions of the image grayscale, the gray level co- occurrence matrix is used to separate the vegetation from the building. The extracted building is made up of a series of polygons that are not smooth. By increasing and removing edges, correct the number of nodes in the polygon and improve the smoothness of the building boundary. In addition, geometry-based regular polygons can be obtained using modeling-based normalization.

(12) Judging the type of change: See Figure 5, the difference between the DSM and the DSM before the change is used to obtain the height change area, and the vegetation height difference area is removed from the height change area by the spectral difference between the vegetation and the building. Get the height change area of the building. The height of the building before the change is obtained by the difference between the DSM before the change and the DEM before the change. If the height of the building before the change is zero, the change type of the corresponding height change area of the building is the area change, if the building before the change If the height is not zero, the change type of the corresponding building height change area is height change.

(13) Statistical analysis of polygons in the changing area of the building: statistical analysis of the polygons in the changing area of the building by Arcgis. Based on the 1:10000 urban building geographic information data provided by the urban residential construction and management department, the high-resolution image was taken by the remote sensor tilt photogrammetry is used as the main updated image data source. Thus, we can update the building stock weekly based on the 1:10000 Building Geographic Information data. According to the standard statistical data of illegal buildings, we can get the building stock weekly update by superimposing and analyzing the same attribute data as last week.

(14) Refer to the building planning approval database of the urban housing construction and management department, obtain the geographical coordinates, name, and construction time of the illegal building: import the urban housing construction bureau building planning approval database in the GIS, and the changing area of the screening Attribute information (geographic coordinates, name, start construction time) is queried.

(15) Quantitative extraction of the height or area of illegal buildings: use geographic information software to obtain the height or area of illegal buildings from the digital surface model, add the DSM file in Arcgis, and then create a new point layer, using the space analysis The Extract Value to Point function extracts the elevation in the DSM to the point. Use the computational geometry of Arcgis to obtain floor space. Then calculate the height of the illegal building by the difference from the elevation of the corresponding ground DEM, and obtain the height and area of the building and the corresponding housing record height and area difference in the urban construction and construction bureau building approval database to obtain the illegal height and area.

(16) Deposited into the illegal building database, and conduct the handheld terminal weekly warning through the illegal building monitoring and early warning server: vectorize the illegal building data of this week and store it in the illegal building database, quantitatively extract the height and area. Compare two weeks of vectorized data, obtaining information on changes in height and area of illegal buildings. Finally, through the early warning server, the 
illegal building week change information is sent to the handheld terminal for early warning of the illegal construction week change.

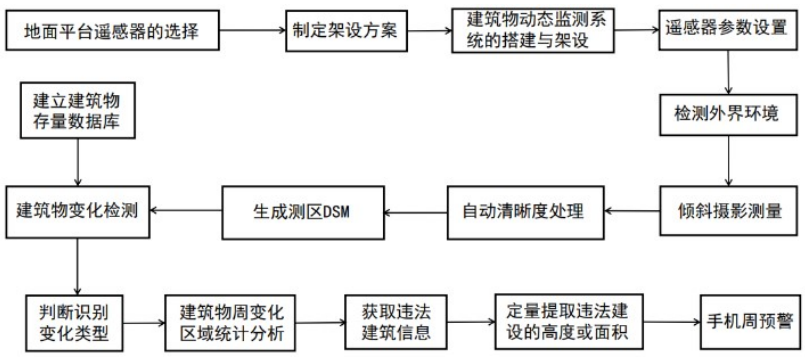

Figure 1. Technical flow chart of the dynamic monitoring method for illegal buildings using large-scale urban terrain to sense big data.

\section{MONITORING SYSTEM}

In order to give full play to the best effect of the method, it is necessary to cooperate with the high point advantage around the survey area, and the optimal erection point of the monitoring system can be analyzed according to the Arcgis visual field. The system satisfies long-term real-time monitoring of urban buildings through tilting photogrammetry based on urban high lying zones. At the same time, the system uses a binocular camera to reduce the number of lenses while ensuring full coverage monitoring of urban buildings, thereby reducing the cost of the camera. In addition, the system adheres to the low-cost, high-quality and intelligent design concept in combination with the actual hardware structure distribution. For example, the temperature and humidity sensor is matched with the camera to install the ambient temperature and humidity around the camera to ensure that the camera captures the image of the area data meets the best conditions for photography, thus ensuring the quality of the collected image data. Secondly, the camera intelligently collects data according to the ambient temperature and humidity parameters fed back by the temperature and humidity sensor, thereby reducing the overall energy consumption.

In addition, the system adopts solar photovoltaic power generation technology to supply power to the tilted camera to solve the problem that the power line erection caused by the conventional power line power supply is difficult, the poweroff correlation is large, and the power supply cost is high The system significantly reduces the workload of manual monitoring, such as realizing real-time warning of illegal buildings, so that illegal building data is sent to the handheld terminal after warning, and Law enforcement agencies can conduct "lightning" enforcement based on illegal building information received. Keep illegal construction in check. The real-time warning of the system avoids the long-term humananalysis process caused by the artificial proactive analysis of most illegal building detection systems, and the illegal buildings can not be discovered in time, which optimizes the monitoring process of illegal buildings and greatly improves the monitoring process. Monitoring efficiency.

\section{CHARACTERISTICS AND INNOVATIONS OF THE MONITORING SYSTEM}

(1) The system makes full use of Spatiotemporal sensor network technology to set up a tilt camera through terrain high point. Based on ArcGIS's visual domain analysis, the optimal observation point is set up with a tilt camera to realize long-term real-time dynamic observation of urban buildings. The temperature and humidity sensor is installed and matched with the tilted camera. The temperature and humidity of the surrounding environment of the tilted camera are detected in real-time, and the data detected in real-time is compared with the measurement condition. When the measurement condition is met, the tilted camera starts to collect data. On the contrary, the data collection work is not performed. The camera working mode based on the temperature and humidity sensor detection data makes the camera work more intelligent and the quality of the data collected by the camera is also guaranteed.

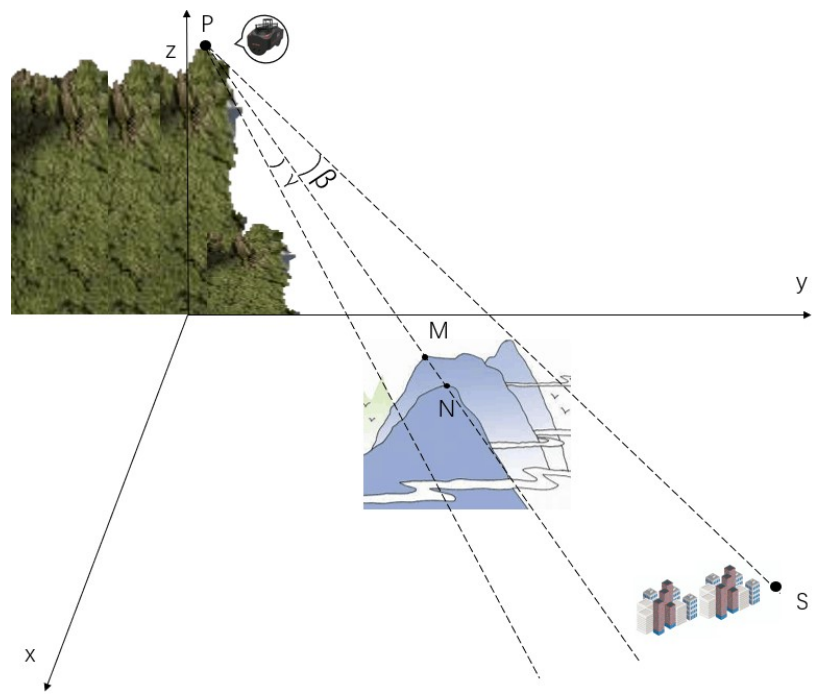

Figure 2. Optimal observation points based on Arcgis visual domain analysis

(2) The system uses solar photovoltaic power generation technology to supply power to the tilted camera. This system uses solar photovoltaic power generation technology to supply power for a tilt photography camera. Compared with the conventional power line power supply method, it solves the problem that power line installation is difficult, costly and prone to associated power failure. Moreover, the solar photovoltaic power generation technology is environmentally friendly, and the system greatly reduces the power supply cost of the camera. More importantly, the solar photovoltaic power generation technology realizes the storage of electric energy in the case of rainy weather.

(3) The system is based on the big data characteristics of the illegal buildings in the real city. The data between the hardware should be transmitted by wireless transmission such as WIFI. For example, the image data collected by the tilted camera of the system is transmitted by WIFI and other wireless transmission methods. The data is transmitted to the spatial analysis server, and the illegal building data obtained by the spatial analysis server is transmitted to the early warning platform through wireless transmission such as WIFI, and the early warning information is also transmitted to the handheld terminal by wireless transmission such as WIFI. Compared with the UAV-based urban illegal building monitoring system, the image data collected by the UAV is stored in the memory card. In this data storage mode, the analyst must insert the memory card into an analysis device 
such as a computer. The image data collected by the manmachine is analyzed and processed, and the system uses the wireless transmission mode such as WIFI to transmit the image data collected by the camera to the spatial analysis server in real-time, and the spatial analysis server can measure the received camera. The real-time analysis and processing of the image data of the area greatly improve the working efficiency of the system; the wireless transmission mode of the data improves the speed of acquiring and transmitting data by the early warning platform, ensuring the timeliness of the early warning. Compared with other urban illegal building monitoring systems, the system improves data transmission efficiency for illegal building monitoring.

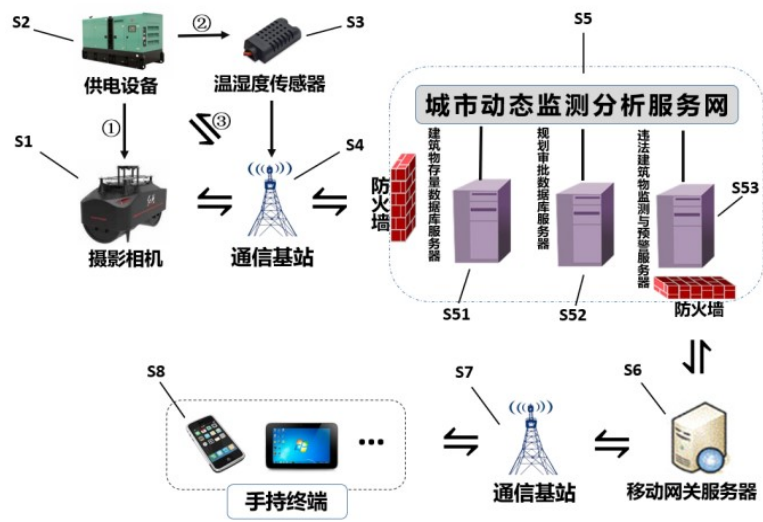

Figure 3. Schematic diagram of data transmission for illegal building dynamic monitoring system

(4) The system monitors buildings with high precision and low cost. For example, the space analysis server generates a high-precision measurement area DSM based on the stereo image pairs collected by the camera, compared with most illegal buildings based on satellite remote sensing images. Besides, remote sensing image data have different cloud coverage levels, and cloud coverage is unpredictable, which may result in an unacceptable quality of captured image data. The system uses the high ground to circumvent this problem and thus makes the collected image data quality high, and at the same time saves the purchase cost of the remote sensing image.

(5) Due to its intelligent nature, the system avoids the shortcomings caused by the artificial proactive analysis of most illegal building monitoring systems, which leads to illegal buildings that cannot be discovered in time. For example, the spatial analysis server in the system detects the change of the ground object based on the three-dimensional incremental analysis in the data processing and uses the multi-scale segmentation algorithm to extract and identify the building and compare the building data with the planning approval data provided by the planning approval database server. Then use ERDAS software to directly obtain the height or area information of the illegal building from the digital surface model (DSM). The data of the type, height, and area of the illegal building change will be sent to the handheld terminal through the warning. It can be seen that the system optimizes the monitoring process of illegal buildings, greatly improves the monitoring efficiency and reduces the labor cost generated by the traditional illegal building monitoring system on the market for analyzing data.
6) The system can pre-warn the illegal building data obtained by the spatial analysis server analysis and processing, and determine whether the actual change degree reaches the early warning level according to the threshold value of the building change degree set by the user, and then decide whether to pass the warning information to the wireless device such as WIFI. The transmission mode is sent to the handheld terminal in real-time for timely warning of illegal buildings. This process is highly automated and significantly reduces the workload of manual monitoring.

(7) The handheld terminal of the system can query the warning information, including the coordinates of the illegal building, the type of illegal construction, the height or area of the illegal construction, etc.; and the handheld terminal has the navigation function, which can plan the best law enforcement path for the law enforcement personnel to make the law enforcement personnel It is possible to reach the coordinates of illegal buildings at the fastest speed, making lightning-type law enforcement possible. In addition, the handheld terminal also has a statistical analysis function, which can count and analyze the number and type of illegal buildings in a certain area. This provides reliable technical support for law enforcement agencies to define key management areas.

\section{CONCLUSION}

The method utilizes the high ground point around the survey area to expand the monitoring range, improve the accuracy of the monitoring data, realize long-term real-time monitoring in the field of illegal building monitoring, generate DSM based on stereo image pairs, and detect the changed features through three-dimensional incremental analysis. Extract and identify buildings using multi-scale segmentation algorithms, precise DEM and DSM, then perform statistical analysis, change type identification, deposit into the database, and finally send illegal building data to handheld terminals through early warning, and provide law enforcement department illegal building information in real-time. This method fully utilizes the characteristics of intelligent, autonomous, and wireless transmission of Spatiotemporal sensor network technology, which significantly reduces the workload of manual monitoring, greatly shortens the process from the emergence and discovery of illegal buildings in cities and improves the efficiency of dynamic monitoring and data entry databases for illegal buildings in cities.

The monitoring system designed according to its principle uses the Spatiotemporal sensor network technology to set up the tilted camera through the terrain high point. The temperature and humidity sensor is matched with the camera to install the ambient temperature and humidity around the camera, ensuring that the camera collects the image data of the survey area. When the optimal conditions for photography are met, the camera operating mode based on the temperature and humidity sensor detection data makes the camera work more intelligent and the quality of the data collected by the camera is also guaranteed. At the same time, solar photovoltaic power generation technology is used to supply power to the tilted camera, which reduces the power supply cost of the camera and realizes energy storage. The data between the various hardware in the system should be transmitted by wireless transmission such as WIFI to 
improve the speed of the early warning platform to acquire and send data and ensure the timeliness of early warning.

\section{ACKNOWLEDGMENTS}

This work was supported by the Guilin Scientific Research and Technology Development Plan(20190601),the Innovation and Entrepreneurship Training Program of Chinese College Students (NO.201910596630); Chongqing basic science and advanced technology research (cstc2015jcyjBX0023). Special thanks to the College of Geomatics and Geoinformation, Guilin University of Technology for the support of our work.

\section{REFERENCES}

Zheng Zhihong, Yang Wenjing, Analysis of Illegally Built Buildings Monitoring Based on UAV Remote Sensing Image, Urban Geotechnical Investigation \& Surveying,2016,(06),84-87

Li Yi, Study on the Existing Problems and Countermeasures of Illegal Buildings in Pingtan, Fujian Agriculture and Forestry University,2017:1-10

Zhu Jianwei, Yuan Guohui, Finding illegal construction in city by using unmanned aerial vehicle based on the tilt photogrammetry, Geotechnical Investigation \& Surveying, 2017,45(07),59-62.

$\mathrm{Yu}$ Chuanfu, Li Junji,2018: Application of Oblique Photogrammetry Technology in Urban Planning, Construction and Management, 2018,38(03),51-56 DOI:10.16169/j.issn.1008-293x.k.2018.09.009.

ZHANG Chunxiao, WEN Gaojin, WANG Hongmin , SHANG Zhiming; Fast Aerial Image Processing for UAV Dynamic Monitoring.[J]Bulletin of Surveying and Mapping,2015(1): $\quad$ 50-54.DOI: $\quad$ 10.13474/j.cnki.112246.2015.0009

ZHOU Jie,2016: Research on the Method of Constructing Realscene 3D Model Based on Oblique Photogrammetry Technology, Faculty of Land Resource Engineering, Kunming University of Science and Technology, Kunming 650093, China, 10.14018/j.cnki.cn13-1085/n.2016.25.089.

Qian Y, Tang L N, Zhao J Z. A review on building height extraction using remote sensing images. Acta Ecologica Sinica,2015, 35 (12):3886-3895.

Licciardi G A, Villa A, Dalla Mura, M, Bruzzone L, Chanussot, J, Benediktsson, J A. Retrieval of the height of buildings from WorldView-2 multi-angular imagery using attribute filters and geometric invariant moments. IEEE Journal of Selected Topics in Applied Earth Observations and Remote Sensing, 2012, 5 (1): 71-79.

Zhao Z M Zhou X C Fu Q K, et al. Method for extraction of building height information based on ZY-3 image [J]. Remote Sensing for Land and Resources,20,27(3):19-24.
Tian X G, Zhang J X, Zhang Y H. Extraction of heights of buildings in city from shadows in QuickBird image [J]. Science of Surveying and Mapping,2008,33(2):88 -89,77.

SUN Jinke, JING Haitao, CAO Dan, CHEN Zhengchao, Using Satellite Stereo Image Extract Urban Plot Ratio, Geomatics \& Spatial Information Technology, 2019,42(04),41-44.

Zheng ZHA, Luo CHEN, Ye WU, Ning JING, 2017: SparkBased Iterative Spatial Overlay Analysis Method.doi.10.2991/eia-17.2017.49.

Zhan Yunjun, Yang Shuwen, 2002: Extracting Buildings Distribution Information of Different Heights from the Shadows in High Resolution Images, Journal of Xianning Teachers College, doi.org/10.16751/j.cnki.hbkj.2002.03.028.

Revised August 2019 